\title{
Peer support networks: A local approach to the global issue of moral injury in surgical training and practice
}

\author{
Sunil K. Geevarghese, MD, MSCl'; Elizabeth A. Pomfret, MD, PhD' \\ 'Vanderbilt Transplant Center, Vanderbilt University Medical Center, Nashville, TN, United States; ${ }^{2}$ Transplant Division, University of Colorado Anschutz Medical Campus, Denver, C0, United States
}

Cite as: Geevarghese SK, Pomfret EA. Peer support networks: A local approach to the issue of moral injury in surgical training and practice. Can Urol Assoc J 2021;15(6Suppl1):S33-5. http://dx.doi.org/10.5489/cuaj.7300

\section{Introduction}

The need is clear. The strain on medical practitioners is unparalleled. The COVID-19 pandemic has been a powerful aggravating factor for moral injury. Much has been written in the lay press and PubMed, including an American Psychiatric Association (APA) guidance document, "Moral injury during the COVID-19 pandemic. ${ }^{\prime 1}$ In it, the APA recommends a three-tiered approach in addressing moral injury by healthcare workers: preventative interventions, support for teams at risk, and identify and address moral injury. Peer support is a powerful way to address all three. In this brief review, we will discuss how peer support systems can provide a local approach to the global problem of moral injury.

\section{What is moral injury?}

Moral injury occurs when clinicians bear witness to or fail to prevent an act that transgresses our moral belief of putting the patient first. For this brief treatment of the complex problem of moral injury, we will limit the term to describe what may happen to surgeons when patients undergo complications during appropriate care. A key point here is that we should not limit discussions of moral injury to what occurs when we err. Major complications, such as anastomotic leaks and intraoperative death, may take place despite providing standard of care treatment. In aggregate, these events can lead to a deep sense of loss and inadequacy. Dr. René Leriche, a French vascular surgeon and physiologist who was a mentor for many surgeons, including Dr. Michael DeBakey, stated in his 1951 book, "La Philosophie de la Chirurgie," : : Every surgeon carries within himself a small cemetery, where from time to time he goes to pray, a place of bitterness and regret where he must look for an explanation for his failures." This poignant statement powerfully encapsulates the intense and ongoing dynamics of moral injury in the life of the surgeon.
Luu et al describe moral injury as it happens in four stages: the kick, the fall, the recovery, and the long-term impact. $^{3}$ They explored surgeon reactions to adverse events and the impact on judgement and decision-making using a constructivist grounded theory approach with surgeon interviews. They describe the kick as a visceral blow, accompanied by feelings of failure, self-doubt, and unworthiness. The fall is characterized by feelings of spiraling out of control. A surgeon's quote taken from the paper that illustrates this well is, "if they develop a complication, even if it wasn't your fault, then you go back to the point where you think, well, maybe I shouldn't even have offered them surgery and what would their life have been like if they didn't have an operation." Getting over the injury often requires the passage of time, the recovery. Harmful ramifications of the recovery include cancelling subsequent similar cases. The long-term impact is marked by the cumulative effect of complications on the surgeon's sense of self. One admitted that "a piece of them [is] being taken away with every complication." The major cost is that moral injury leads some to change practice or seek early retirement. For those who continue to practice surgery, moral injury can cloud judgement and lead to risk-averse behavior due to heightened scrutiny by subordinates, peers, and administrators.

\section{Second victim syndrome}

Unmitigated moral injury over time may lead to "second victim syndrome" (SVS), which is accompanied by feelings of responsibility for unexpected patient outcomes, failing their patients, second-guessing their clinical skills and knowledge base, declining status in the surgical community, and humiliation that persists for years. Scott et al delineate six dynamic stages of the SVS. ${ }^{4}$ The first stage is the chaos and accident response, which is notable for distracting self-questioning while caring for other similar patients. The intrusive reflections stage is marked by preoccupation by repetitive replays of the event, which leads to isolation and a loss of confidence. Restoring personal integrity is the third stage and describes the process of re-integration into professional life with the help of a mentor. Enduring the inquisition reflects 
the process of scrutiny that the event entails by the institution and it leads to increased anxiety. Obtaining emotional first aid is the fifth and critical step that is unfortunately overlooked and is a need that peer support can fill. Moving on is the sixth and final step and sadly, is often a literal moving away due to loss of reputation and employment.

Wu et al reflect on the term "second victim" as complicated and associated with negative connotations for both the clinician and the "first victim." It also seems a poor choice because it more commonly refers to a devastating loss resulting from the deliberate intention of the perpetrator. Victim can also convey passivity and helplessness. On the other hand, it is an established term for nearly two decades by the National Quality Forum Safe Practices for Healthcare and by the Joint Commission. Proponents of this term claim that second victim seizes attention of healthcare workers, institutional leaders, and policy makers and acknowledges the trauma of the suffering clinician. It is for the latter two ideas that we continue to use this term in this manuscript.

\section{Focus on transplant surgeons}

The pressure on transplant surgeons is even greater due to the profound nature of their patients' illness and the intensely physical and mental work of the operation (Table 1 includes specific descriptions of moral injury by transplant surgeons). During training, transplant surgeons can be shielded from the pressures of moral injury by their faculty. As trainees emerge into independent practice at the end of transplant surgical fellowship, they are granted the muchdesired independence in surgical decision-making and operating. Often, the newly minted surgeon is, for the first time, facing major patient mortality and morbidity while trying to establish their own ability, availability, and affability, colloquially termed "the 3 As." These aspects establish their fragile reputation as they face moral injury and is often an isolating experience for young surgeons, generating a

\footnotetext{
Table 1. Moral injury in their own words

"Several complications over a short time frame left me defeated and questioning the competencies I've been honing for nearly a decade."

"When my patient died on the table because of my technical error, I was silently, secretly distraught for months."

"I had some complications that preoccupied my mind early in my career and I questioned my skills. I did not have many people to reach out to, and it took some time to grow professionally with bumps along the road. I would have benefitted from a frank and supportive discussion with a peer."
}

fertile ground for self-questioning and second-guessing, which together can potentiate the impact of moral injury. Although well-meaning theoretically and important from a quality assurance/process improvement, morbidity and mortality (M\&M) conferences can play a highly detrimental role in the recovery of the injured. It requires, after time has passed but often before healing has occurred, a reliving of the details of the event while preparing for the presentation. At the podium, the surgeon faces isolation and humiliation, which can occur in front of others who are more junior to them. Seemingly unavoidable, the rigors of $M \& M$ are a rite of passage in most surgical training programs.

\section{Additional factors}

Other factors that lower the threshold for moral injury include litigation from medical malpractice claims. This element is particularly damaging if it occurs in the first years of practice and if the surgeon is left to defend himself/herself rather than having a practice or hospital risk management team involved. As moral injury is only one contributor to burnout, the coupling of other issues, such as marital discord/divorce, as well as physical and mental health changes, can accelerate the transition to burnout.

\section{Peer support network}

A peer support network is a well-established tool that systems can use to support their surgeons in training or practice. Shapiro and colleagues established the first major hospital-based unit in the United States, the Center for Professionalism and Peer Support (CPPE) at the Brigham and Women's Hospital in Boston, MA. ${ }^{6}$ Realizing that surgeons rarely access available supports and intrinsically appear reluctant to acknowledge self-perceived weakness, they created an outward-facing system where peers provide added support on top of established employee assistance personnel (EAP). Members of the CPPE were trained by the EAP. Combined with personal perspectives on their own experiences with moral injury, these peer supporters were able to provide "at the elbow" guidance in real time and mobilize resources to the injured individual without relying on the surgeon to reach out. Matching between peers was attempted in broad strokes to include parameters such as specialty, years in practice, and personality style. However, great care was taken to avoid matching the injured individual with those to whom they report. ${ }^{7}$

The American Society of Transplant Surgeons (ASTS) set out to create the first specialty society-based peer support network for its members as a benefit. The authors aligned ASTS members across the United States and Canada with 
the goal to provide support to members and trainees who have suffered an adverse event. Each member of the ASTS Peer Support Network underwent training by Dr. Shapiro in August 2020 founded on key principles (Table 2).

\section{How does the ASTS Peer Support Network work?}

Referrals can be submitted anonymously online via asts.org or by phone. Minimal information (name, best contact method, and brief description of reason for referral) is required. All information is kept secure and confidential and not revealed to employers or other entities. Information is then screened by ASTS staff and the senior author and distributed to appropriate members of the Network, matched in a similar way to the CPPE. After the Network member reaches out to the individual, there is a followup offering them specific resources as desired. Additional outreach occurs at one week after contact by the preferred contact method as a collegial check-in. The main goal of the Network is to provide emotional first aid to the affected member. After the initial contact, resources can be mobilized to their aid, including formal counselling.

The ASTS Peer Support Network is a new program that provides much needed counsel for the trainee or practicing transplant surgeon in the midst of moral injury or other sources of personal or professional strife. To our knowledge, this may be one of the first medical specialty society-based peer support networks. Launching this network within our specialty allows us to leverage a common bond that all members share: single training transplant pathway and several elements of practice that are universal across the spectrum of practices (e.g., severely ill transplant recipients, complex donor procurements, challenging operations, and major patient morbidity). This allows both Network member and participant the opportunity to connect on these common elements. As Network members have had their own experiences with moral injury, they are often willing to share their pitfalls and transformational moments to commiserate and provide inspiration. We anticipate the system will become a commonly used resource as we continue to destigmatize moral injury. The ASTS has developed a de-identified database to record basic demographic information of the members accessing this resource that will hopefully aide in assessing the utilization of the program. The ASTS Peer Support Network is

\begin{tabular}{l}
\hline Table 2. Peer support principles \\
\hline Loving presence \\
Psychological safety \\
Empathic listening \\
Problem solving guidance \\
Reframing \\
Appreciation \\
Coping mechanisms \\
Resource connection \\
Non-judgemental curiosity \\
\hline
\end{tabular}

also creating a short survey to be distributed to users after intervention to determine the benefits of this initiative.

\section{Conclusions}

The ASTS Peer Support Network promises to augment existing support within hospital systems. Connecting with peers outside of their institution may allow greater confidentiality than hospital offerings, such as EAP. Although early in its genesis, this Network's approach of using common training and practice to connect members can be employed by other societies or groups as well. A fundamental principle of the Network is an interest in others while being willing to show vulnerability to your peers. For these reasons, we believe that society-based peer support is a unique opportunity to address the problem of moral injury in surgical training and practice.

\section{References}

1. American Psychiatric Association. COVID-19 pandemic guidance document: Moral injury during the COVID-19 pandemic, 2020

2. Leriche R. [La Philosophie de la Chirugie]. Prog Med (Paris) 1950;78:475-83.

3. Luu S, Patel P, Laurent St-Martin ASL, et al. Waking up the next morning: Surgeon's emotional reactions to adverse events. Med Educ 2012;46:1 179-88. https://doi.org/10.1111/medu. 12058

4. Scott SD, Hirschinger LR, Cox KR, et al. The natural history of recovery for the healthcare provider "second victim" after adverse patient events. Qual Saf Health Care 2009;18:325-30. https://doi.org/10.1136/qshc.2009.032870

5. Wu AW, Shapiro J, Harrison R, et al. The impact of adverse events on clinicians: What's in a name? J Patient Saf 2017;16:65-72. https://doi.org/10.1097/PTS.0000000000000256

6. Shapiro J, Galowitz P. Peer support for clinicians: A programmatic approach. Acad Med 2016;91:12004. https://doi.org/10.1097/ACM.0000000000001297

7. Hu Y-Y, Fix ML, Hevelone ND, et al. Physicians' needs in coping with emotional stressors: The case for peer support. Arch Surg 2012;147:212-7. https://doi.org/10.1001/archsurg.2011.312

Correspondence: Dr. Sunil K. Geevarghese, Vanderbilt University Medical Center, Nashville, TN, United States; s.geevarghese@vumc.org 